\title{
SUR LA REPRODUCTION ET LA PONTE \\ D'ARMIGERES OBTURBANS WALKER AU TONKIN
}

\author{
Par Henri GALLIARD
}

Armigeres obturbans est très répandu en Extrême-Orient. C'est au Tonkin, à Hanoï particulièrement, un culicidé strictement domestique, extrêmement agressif pour l'homme pendant la saison chaude. Nous avons pu nous en procurer une souche au mois d'octobre dernier et notre élevage en est à sa sixième génération. Il nous a paru intéressant de signaler quelques particularités de sa reproduction et de sa ponte.

Accouplement. - Cette espèce, sous forme d'adulte, disparaît complètement pendant toute la saison froide. Il est done naturel de penser que l'accouplement ne puisse se faire qu'à une température relativement élevée. Mais au laboratoire, dans un climat donné, on peut constater que toutes les espèces peuvent se reproduire à peu près aux mêmes températures. En particulier, pour A. obturbans, l'accouplement se fait à partir de $20^{\circ}$, alors que dans les conditions naturelles, on ne rencontre jamais ni mâles, ni femelles à cette température, et cesse à partir de $33^{\circ}$.

Les travaux de Roubaud ont montré que certaines espèces ou races d'une même espèce de culicidés pouvaient se différencier par leur faculté de se reproduire en espace restreint (sténogamie). A. obturbans possède, à un degré remarquable, cette propriété, puisque nous avons pu obtenir une fertilisation dans les cages de Missiroli mesurant $8 \mathrm{~cm} . \times 8 \mathrm{~cm} . \times 10 \mathrm{~cm}$. L'accouplement se produit indifféremment avant ou après le repas de sang.

Ponte. - Un repas de sang est nécessaire pour assurer la ponte. Malgré tous nos essais, nous n'avons pu obtenir ce que Roubaud a appelé le "cycle autogène ». La ponte se fait dans les délais normaux, c'est-à-dire au bout de 48 heures, à une température optima de $28^{\circ}$.

Mode de ponte. - Dans les conditions naturelles, malgré la quantité innombrable d'adultes envahissant les habitations au mois d'octobre et novembre, nous n'avons jamais pú déterminer la

Annales de Parasitglogie, T. XIV, ${ }^{\circ} 5 .-1^{\text {er }}$ septembre 1936. 
ponte d'Armigeres obturbans dans aucun récipient, alors qu'il est si facile de le faire pour Aedes (Stegomyia) albopictus, A. argenteus et $C$. fatigans. Les larves vivent uniquement dans les eaux très polluées, soit dans les fosses, soit dans les égouts en plein air, alors que $C$. fatigans est beaucoup plus ubiquiste.

Expérimentalement, la ponte peut se faire de plusieurs façons; mais dans la majorité des cas, les femelles pondent comme les Stcgomyia sur les parois du récipient, presqu'au ras de la surface de l'eau. De plus, les œufs ne sont pas aussi dispersés, soit en hauteur, soit en largeur, que dans le cas de Stegomyia; mais ils forment le plus souvent un anneau régulier à quelques millimètres au-dessus de la surface de l'eau. Les œuf's sont rapprochés les uns des autres, parfois fortement adhérents en masse compacte que l'on peut décoller tout d'un bloc.

La femelle pond même de préférence sur des tiges diverses ou de simples morceaux de bois placés dans les bocaux. Les oufs sont alors orientés tout à fait irrégulièrement et tapissent entièrement la surface du support sur une certaine hauteur. C'est probablement le mode de ponte qui doit se rencontrer le plus fréquemment dans la nature.

Dans nos élevages, nous avons également réussi facilement à faire pondre les femelles sur du coton humide et directement à la surface de l'eau.

Les substances contenues dans l'eau et qui servent à l'alimentation des larves, que ce soit de la poudre de rate ou de foie, de la poudre de larves, de la pulpe de banane, des déjections de cobaye, de chien ou de chat, ne semblent pas exercer une attraction plus grande que l'eau distillée sur les femelles gravides, dans les conditions expérimentales.

Intensité de ponte. - Le nombre des œufs pondus est variable dans une certaine mesure avec chaque spécimen, variable surtout suivant les conditions dans lesquelles la femelle pond; et enfin, dans un élevage au laboratoire, le nombre des œufs subit également des variations importantes chez les générations successives.

Une femelle capturée, fécondée et gorgée, pond en moyenne, sur les bords du verre, 110 oeufs. Dans un cas, une femelle a pondu 180 œufs en une seule fois.

Sur le coton humide, une femelle pond en moyenne 65 oufs, sur l'eau 90 oufs.

Les femelles pondent quelquefois d'abord sur la paroi puis achèvent leur ponte sur l'eau où elles déposent à peu près 10 pour 100 de leurs œufs. 
Lorsque les conditions sont défavorables, particulièrement quand la femelle se trouve en espace trop restreint, la ponte est souvent faible. Ainsi, dans les cages de Missiroli, le nombre des oufs n'a jamais dépassé 40, que la femelle meure après ou non.

Ainsi le fait que la femelle pond ses œufs surtout au-dessus de la surface de l'eau, dans les égouts, permet d'expliquer l'apparition très tardive des adultes de cette espèce, au moment des premières pluies importantes. Nous avons capturé, en effet, en 1936, la première femelle le 12 mai.

Les femelles d'Armigeres obturbans peuvent-elles pondre plusieurs fois? Elles meurent parfois après la première ponte, même quand les conditions semblent favorables. Mais elles peuvent souvent pondre une deuxième fois, après un nouveau repas. Nous n'avons jamais pu obtenir de troisième ponte. Le nombre des cuffs de la deuxième est toujours très faible et il n'est pas proportionnel à celui de la première.

Ainsi, une femelle ayant pondu 180 oufs sur la paroi, pond, deux jours après un nouveau repas de sang, 14 oufs sur l'eau et meurt. Une autre femelle, dans une cage de Missiroli, pond 30 œufs sur l'eau, puis 8 œufs après un deuxième repas et meurt.

Nous avons constaté également, pour un élevage donné (Exp. 14), une diminution assez sensible du nombre des oufs chez les femelles des diverses générations. C'est ainsi que la femelle capturée dans la nature a pondu 120 oufs, les femelles de la deuxième génération ont pondu en moyenne 130 œufs, celles de la troisième génération 90 , la quatrième 110 , la cinquième 60 et la sixième 35 . Quant à la septième génération, une seule femelle a consenti à pondre 18 oufs.

Influence de l'alimentation des larves sur la ponte. - Nous avons cherché à savoir si la nature des substances alimentaires fournies aux larves avait une influence quelconque sur la fécondité des femelles. Expérimentalement, les larves d'Armigeres obturbans arrivent à un complet développement et dans le même temps, quand on leur fournit soit de la poudre de rate ou de poisson séché, soit de la pulpe de banane. Dans ces conditions, en utilisant des larves de la première génération, nous pouvons affirmer que les femelles nées de ces larves se sont comportées de façon absolument identique.

Il n'en est pas de même lorsque les larves sont nourries de déjections de cobaye, par exemple. Leur développement est infiniment plus long et reste incomplet. Les adultes sont plus petits et 
le nombre des oufs pondus est alors beaucoup moins élevé ; il est de 70 au maximum contre 120 en moyenne dans les deux premiers cas.

\section{RÉSUMÉ}

Dans cette note, nous apportons quelques observations, dans les conditions expérimentales, sur la ponte d'Armigeres obturbans à Hanoï. L'intensité de la ponte est essentiellement variable suivant les conditions plus ou moins favorables dans lesquelles se trouvent les femelles (espace, lieu de ponte, nutrition des larves). La ponte des œufs au-dessus de la surface de l'eau permet d'expliquer l'apparition très tardive des adultes de cette espèce, à Hanoï, au moment des premières grandes pluies.

Laboratoire de Parasitologie de l'Ecole de Médecine de l'Indochine (Hanö̈, Tonkin). (Directeur : Prof. H. Galliard). 